\title{
Concurrent Testing of Droplet-Based Microfluidic Systems for Multiplexed Biomedical Assays*
}

\author{
Fei Su, Sule Ozev and Krishnendu Chakrabarty \\ Department of Electrical \& Computer Engineering \\ Duke University, Durham, NC 27708 \\ E-mail: \{fs, sule, krish\}@ee.duke.edu
}

\begin{abstract}
We present a concurrent testing methodology for detecting catastrophic faults in droplet-based microfluidic systems and investigate the related problems of test planning and resource optimization. We apply this methodology to a droplet-based microfluidic array that was fabricated and used to perform multiplexed glucose and lactate assays. The test approach interleaves test application with the biomedical assays and prevents resource conflicts. We show that an integer linear programming model can be used to minimize testing time for a given hardware overhead due to droplet dispensing sources and capacitive sensing circuitry. The proposed approach is therefore directed at ensuring high reliability and availability of bio-MEMS and lab-on-a-chip systems, as they are increasingly deployed for safety-critical applications.
\end{abstract}

\section{Introduction}

Microfluidics-based miniaturized systems for biochemical analysis have become popular in recent years $[1,2,3]$. These composite microsystems, also known as bio-MEMS or lab-on-a-chip (LoC), offer several advantages over macroscopic systems, such as design flexibility, smaller size, lower cost, and higher sensitivity. They enable controlling small amounts (e.g., nanoliters) of fluids, thus reducing sample size and reagent volume as well as power consumption. Advances in microfluidic techniques offer exciting possibilities in the realm of massively-parallel DNA analysis, automated drug synthesis, and real-time biomolecular detection and recognition.

Clinical diagnosis is one of the most promising applications for these techniques $[4,5]$. The benefits of miniaturization in microfluidics offer immediate point-ofcare diagnosis of diseases. Techniques to counter bioterrorism can also benefit from microfluidics $[6,7,8]$. Such microfluidics-based systems, capable of continuous sampling and real-time testing of air/water samples for biochemical toxins and other pathogens, can serve as an always-on "bio-smoke alarm" to offer an early warning

* This research was supported in part by the National Science Foundation under grant number EIA-0312352 capability to combatants or citizens.

Currently, most microfluidic systems, consisting of micro-pumps, micro-valves, and micro-channels, are based on the principle of continuous fluid flow $[9,10]$. A promising alternative paradigm involves the manipulation of liquids as discrete microdroplets [11, 12]. Dropletbased microfluidic systems enable easy reconfigurability since each droplet can be controlled independently and each cell in the array has the same structure. The feasibility of performing real-time biomedical assays, e.g., the colorimetric enzyme-kinetic glucose assay or the polymerase chain reaction (PCR), on these novel microsystems has recently been demonstrated experimentally [13, 14, 15].

As droplet-based microfluidic systems are widely deployed in safety-critical biomedical applications, the reliability of these systems has emerged as a critical performance parameter. These systems need to be tested adequately not only after fabrication, but also continuously during in-field operation. For instance, for detectors monitoring for dangerous pathogens in critical locations such as airports, field testing is critical to ensure low false-positive and false-negative detection rates. In such cases, concurrent testing, which allows testing and normal biomedical assays to run simultaneously on a microfluidic system, can play an important role. It consequently facilitates built-in self-test (BIST) of microfluidic systems and makes them less dependent on costly manual maintenance on a regular basis.

Next-generation system-on-chip designs are expected to be composite microsystems incorporated with microelectromechanical and microfluidic components. Therefore, there is a need for efficient testing methodologies for these microsystems. The ITRS 2001 document recognizes the need for new test methods for disruptive device technologies that underly microelectromechanical systems and sensors, and highlights it as one of the five difficult test challenges beyond 2007 [16].

In [17], a fault model and a unified test methodology for droplet-based microfluidic systems are presented. The authors classify physical defects in such systems as either catastrophic or parametric, and illustrate how faults can be 
detected by controlling and tracking droplet motion electrostatically. This cost-effective test methodology facilitates concurrent testing for droplet-based microfluidic systems. Test planning and resource optimization are motivated by the need for concurrent testing.

In this paper, we present a concurrent testing methodology for detecting catastrophic faults in dropletbased microfluidic systems and investigate the problems of test planning and resource optimization. An integer linear programming (ILP) model is formulated to derive an optimal droplet flow path for concurrent testing. We apply this methodology to a droplet-based microfluidic system performing multiplexed biomedical assays, and develop a concurrent test plan for this system.

The organization of the remainder of the paper is as follows. In Section 2, we present an overview of dropletbased microfluidic systems and a multiplexed biomedical assay that can be performed using such systems. Next, a concurrent testing methodology to facilitate in-field monitoring is discussed in Section 3. Related prior work is discussed in Section 4. Section 5 presents an integer linear programming (ILP) model based on the notion of scheduling using time-slots. We minimize the test application time for a given hardware overhead by optimizing the test plan using the ILP model. Section 6 evaluates this concurrent testing methodology by applying it to a droplet-based microfluidic system used for multiplexed biomedical assays. Finally, conclusions are drawn in Section 7.

\section{Droplet-Based Microfluidic Systems and Multiplexed Biomedical Assays}

The microfluidic system discussed in this paper is based on the manipulation of nanoliter droplets using the principle of electrowetting. Electrowetting refers to the modulation of the interfacial tension between a conductive fluid and a solid electrode by applying an electric field between them. By varying the electrical potential along a linear array of electrodes, electrowetting can be used to move nanoliter volume liquid droplets along this line of electrodes [11]. Droplets can also be transported, in userdefined patterns and under clocked-voltage control, over a two-dimensional array of electrodes without the need for micropumps and microvalves.

The advantages of reduced reagent consumption, simplicity of sensing, and rapid analysis facilitate the widespread deployment of droplet-based microfluidic systems in a clinical point-of-care setting. The in-vitro measurement of glucose and other metabolites, such as lactate, glutamate and pyruvate, is of great importance in clinical diagnosis of metabolic disorders. Recently, a colorimetric enzyme-kinetic glucose assay performed on the droplet-based microfluidic system has been successfully demonstrated $[13,14,18]$. This system uses a basic microfluidic platform, which moves and mixes droplets containing biomedical samples and reagents, and an integrated optical detection system consisting of a LED and a photodiode; see Figure $1[13,14]$.

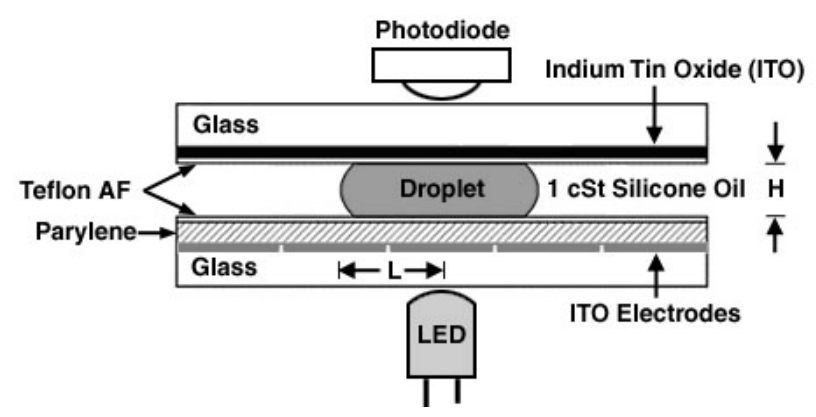

Figure 1: Droplet-based microfluidic systems used for colorimetric enzyme-kinetic assays.

The basic cell of a droplet-based microfluidic system consists of two plates, and the filler medium, i.e., the silicone oil, sandwiched between the plates. The droplets travel inside the filler medium. The bottom plate contains a patterned array of individually controllable electrodes, and the top plate is coated with a continuous ground electrode. All electrodes are formed by optically transparent indium tin oxide (ITO). An 800-nm-thick dielectric insulator, i.e. Parylene C, coated with a $60-\mathrm{nm}$ thick hydrophobic film of Teflon AF, is added to the top and bottom plates to decrease the wettability of the surface and to add capacitance between the droplet and the control electrode. The length of the control electrode $\mathrm{L}$ is $1.5 \mathrm{~mm}$ and the height between two plates $\mathrm{H}$ is $0.475 \mathrm{~mm}$ [13]. The detailed fabrication process is described in [19]. The basic principle of droplet transportation is to electrostatically control the interfacial tension at the droplet/insulator interface. A control voltage is applied to an electrode adjacent to the droplet and at the same time the electrode just under the droplet is deactivated. This causes an accumulation of charge in the droplet/insulator interface, resulting in a surface tension gradient across the gap between the adjacent electrodes, which consequently causes the transportation of the droplet. The velocity of the droplet can be controlled by adjusting the control voltage $(0 \sim 90 \mathrm{~V})$, and droplets can be moved at speeds of up to $20 \mathrm{~cm} / \mathrm{s}$. Based on this principle, microfluidic droplets can be moved freely to any location of a twodimensional array. In the glucose assay experiment, the actuation voltage was set at $50 \mathrm{~V}$ [13].

The glucose assay performed on the droplet-based microfluidic system is based on Trinder's reaction, a colorimetric enzyme-based method. The enzymatic reactions involved in the assay are:

$$
\begin{gathered}
\text { Glucose }+\mathrm{H}_{2} \mathrm{O}+\mathrm{O}_{2} \stackrel{\text { GlucoseOxidase }}{\longrightarrow} \text { GluconicAcid }+\mathrm{H}_{2} \mathrm{O}_{2} \\
2 \mathrm{H}_{2} \mathrm{O}_{2}+4-\mathrm{AAP}+\mathrm{TOPS} \stackrel{\text { Peroxidase }}{\longrightarrow} \text { Quinoneimine }+4 \mathrm{H}_{2} \mathrm{O}
\end{gathered}
$$




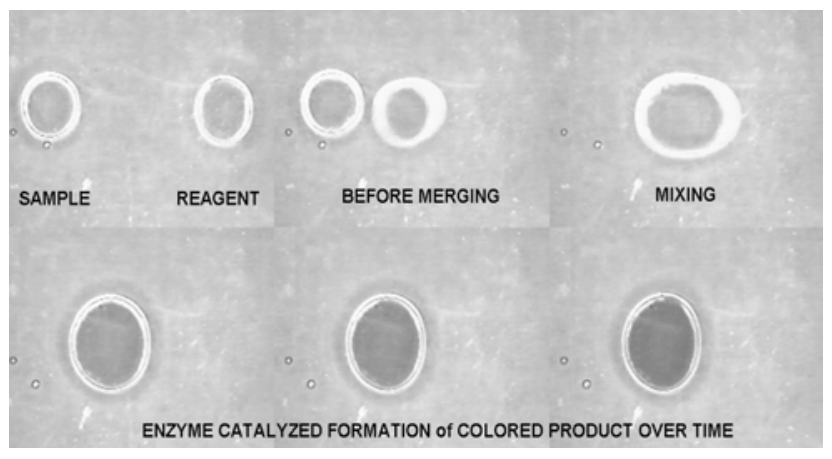

Figure 2: Photos in different steps of glucose assay.

In the presence of Glucose oxidase, glucose can be enzymatically oxidized to gluconic acid and hydrogen peroxide. Then, in the presence of peroxidase, the hydrogen peroxide reacts with 4-amino antipyrine (4AAP) and N-ethyl-N-sulfopropyl-m-toluidine (TOPS) to form violet-colored quinoneimine, which has an absorbance peak at $545 \mathrm{~nm}$. Based on this colorimetric reaction, the complete glucose assay can be performed following three steps, namely, transportation, mixing and optical detection; see Figure 2 [18]. Sample droplets containing glucose and reagent droplets containing glucose oxidase, peroxidase, 4-AAP and TOPS, are dispensed into the microfluidic system from droplet sources. They are then transported towards a mixer where droplets of the sample and the reagent are mixed together and the enzymatic reaction happens during the mixing. A droplet of the product after mixing is moved to the location of optical detection. The optical detection is performed using a green LED and a photodiode. The glucose concentration can be detected from the absorbance, which is related to the concentration of colored quinoneimine, using a kinetic method. In the kinetic method, the concentration of the glucose is calculated from the rate of the reaction, which is equivalent to the rate of the change of absorbance. The details of the kinetic method for optical detection can be found in [13]. Experiments have shown that the results from the droplet-based microfluidic system match well with the reference values obtained from the conventional measurement $[13,14]$.

In addition to glucose assays, the detections of other metabolites such as lactate, glutamate and pyruvate in a droplet-based microfluidic system have also been demonstrated recently $[13,14]$. Using similar enzymatic reactions and modified reagents, these assays as well as the glucose assay can be integrated to perform a multiplexed biomedical assay concurrently on a dropletbased microfluidic system. Figure 3 shows an image of such a fabricated microfluidic system used for multiplexed biomedical assays. Sample1 contains glucose and

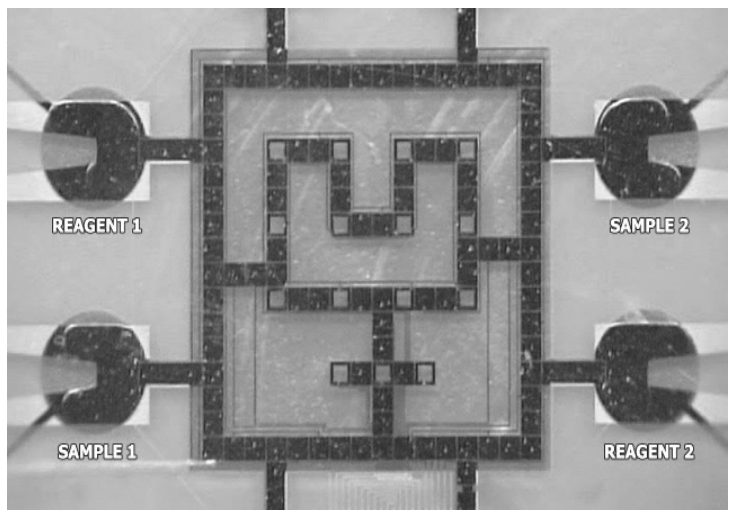

Figure 3: Fabricated microfluidic array used in multiplexed biomedical assays.

Reagent1 contains glucose oxidase and other chemicals. Similarly, Sample 2 contains lactate and Reagent 2 consists of lactate oxidase and other chemicals. To demonstrate multiplexed assays, only cells and electrodes used for the biomedical assay have been fabricated.

\section{Related Prior Work}

Over the past decade, the focus in testing research has broadened from logic and memory test to include the testing of analog and mixed-signal circuits. MEMS is a relatively young field compared to IC design, and MEMS testing is still in its infancy. Recently, fault modeling and fault simulation in surface micromachined MEMS has received attention [20]. In [21, 22], a comprehensive testing methodology for a class of MEMS known as surface micromachined sensors is presented.

However, test techniques for MEMS cannot be directly applied to microfluidic systems, since their actuation mechanism do not handle fluids. Most recent work in this area has been limited to the testing of continuous-flow microfluidic systems. In [23, 24], mixed-signal testing techniques are applied to the problem of testing a microanalysis system. Also, a design-for-testability (DFT) technique for Flow-FET-based microfluidic systems has been proposed [25]. Similar to MOSFET, a Flow-FET has source and drain electrodes over which a relatively large voltage $(\sim 100 \mathrm{~V})$ is applied. Due to the principle of electro-osmotic flow, the electric field moves the charge accumulated between the fluid and the surface of channel, dragging the bulk liquid through the channel.

Electrostatically-actuated, droplet-flow microfluidic systems have recently been proposed as an alternative to continuous-flow systems. However, very limited work on the testing for droplet-based microfluidic system has been reported to date. 


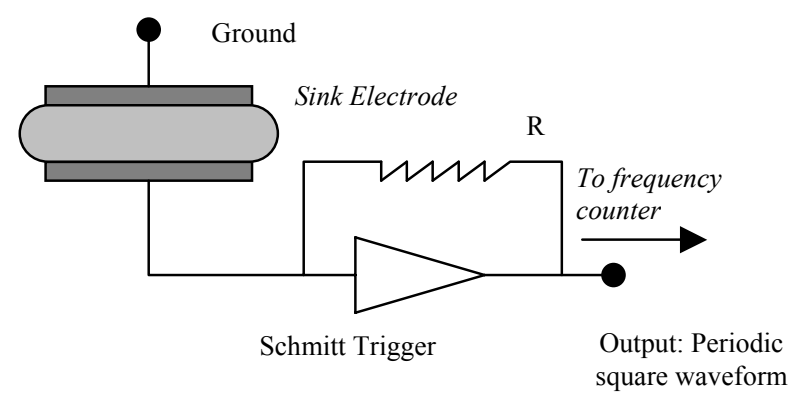

Figure 4: Simple capacitive sensing circuit.

\section{Concurrent Testing Methodology}

Based on the unified detection mechanism proposed in [17], we dispense the test stimuli droplet containing the normal conducting fluid (e.g., KCL solution) into the microfluidic system-under-test from the droplet source. These droplets are guided through the cells following the test plan towards the droplet sink, which is connected to an integrated capacitive detection circuit. An example of such a capacitive circuit is shown on Figure 4. Most catastrophic faults result in a complete cessation of droplet transportation [17]. Thus, for the faulty system, the test stimuli droplet is stuck during its motion. On the other hand, for the fault-free system, all the test stimuli droplets can be observed at the droplet sink by the capacitive detection circuit. Therefore, we can easily determine the fault-free or faulty status of the droplet-based microfluidic system by simply observing the arrival of test stimuli droplets at some selected ports of the system.

This cost-effective fault testing procedure can be performed simultaneously with a normal biomedical assay on a microfluidic system. The problems of test planning and test resource optimization are motivated by the need of the concurrent testing.

The goals and constraints of the test planning and optimization problem are listed as follows:

(1) Concurrency: The test plan should ensure simultaneous executions of testing and biomedical assays and no conflicts between them.

(2) Optimization: There exists an inherent tradeoff between hardware overhead and test application time. Here the hardware overhead is measured by the number of droplet sources and droplet sinks for test application. Test application time should be minimized for a given hardware overhead.

(3) Full coverage: All cells in the microfluidic array, which are not in use for biomedical assays, are considered to be available for testing. The test plan should cover all available cells in the microfluidic array.
The proposed concurrent testing methodology can be used for field-testing of droplet-based microfluidic systems; as a result, it increases the system reliability during everyday operation. With negligible hardware overhead, this method also offers an opportunity to implement BIST for microfluidic systems and therefore eliminates the need for costly, bulky, and expensive external test equipment. Furthermore, after detection, droplet flow paths for biomedical assays can be reconfigured dynamically such that faulty cells are bypassed without interrupting the normal operation. Thus, this approach increases fault-tolerance and system lifetime when such systems are deployed for safety-critical applications.

\section{Optimal Scheduling for Concurrent Testing}

In this section, we formulate the problem of test planning for concurrent testing. The key idea underlying this optimization method is based on the notion of time slots. In order to determine the droplet flow paths, we divide the total test time into equal-length time slots. The length of a time slot equals the time during which a test stimuli droplet moves from a cell to an adjacent cell. The goal of the optimal scheduling problem (OSP) developed in this section is to determine all the time slots at which the microfluidic cells are visited by the test stimuli droplets, such that the total time cost i.e., the time slot at which stimuli droplets reach the sinks after visiting all cells in the array, is minimized.

Although the optimal scheduling problem has been proven to be NP-hard [26], we show that this problem can be solved exactly for a microfluidic array of modest size using integer linear programming (ILP) model. An ILP model can be described as follows:

Minimize: $\boldsymbol{A x} \quad$ (objective function)

Subject to: $\boldsymbol{B} x \leq \boldsymbol{C} \quad$ (constraint inequalities),

where $x$ is a vector of variables, $\boldsymbol{A}$ is an objective function vector, $\boldsymbol{B}$ is a constraint matrix and $\boldsymbol{C}$ is a column vector of constraints. We used a popular public domain ILP solver called lpsolve for our work [27].

Here we use the example of Figure 5 as an illustration to formulate this ILP model. All white cells represent the cells not used by the biomedical assay and therefore available for testing. The black cells are in use by a biomedical application such as droplet mixing, therefore temporarily unavailable for testing. Every cell is represented by two-dimensional coordinates $(i, j)$, where $i$ is the row number and $j$ is the column number of the cell; see Figure 5. 


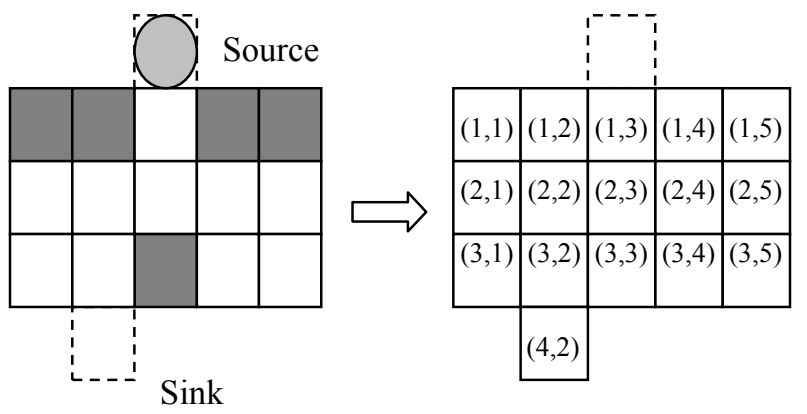

Figure 5: Coordinate representation of an array of cells.

Let $X_{i j k}$ be a binary variable defined as follows:

$X_{i j k}= \begin{cases}1 & \begin{array}{l}\text { if cell }(i, j) \text { is visited by a test stimuli } \\ \text { droplet at time slot } k\end{array} \\ 0 & \text { otherwise }\end{cases}$

where $1 \leq k \leq T$. The parameter $T$ is the maximum possible index for a time slot and its value can be set to an easily-determined loose upper bound.

In the example of Figure 5, cell $(4,2)$ is a sink. Since the sink should be visited exactly once during test application, $\sum_{k=1}^{T} X_{42 k}=1$. Thus, the time slot at which a test stimuli droplet reaches this sink, i.e., all testing operations have been finished, is $C=\sum_{k=1}^{T} k \times X_{42 k}$.

Hence the objective function of the ILP model for OSP is: minimize: $C=\sum_{k=1}^{T} k \times X_{42 k}$.

The following constraint inequalities need to be incorporated into this model.

(1) Testing requirement:

a) $\sum_{k=1}^{T} X_{i j k} \geq 1$ for $(i, j) \in\{A T$ : set of cells available for testing $\}$, i.e., any cell $(i, j)$ in the array available for testing should be visited by the test stimuli droplet at least once.

b) $\sum_{k=1}^{T} X_{i j k}=0$ for $(i, j) \in\{N A T$ : set of cells not available for testing , i.e., any cell $(i, j)$ in array that is running biomedical assays cannot be visited by the test stimuli droplet.

c) $\sum_{k=1}^{T} X_{42 k}=1$, i.e., the sink (the cell $(4,2)$ in here) should be visited by the test stimuli droplet exactly once.

(2) Resource constraint:

Before the test stimuli droplet reaches the sink, only one cell in the array can be visited by this droplet in any time-slot. After that, no cells in the array (including the sink) can be visited by a test stimuli droplet again. This constraint for an $m \times n$ array (a $3 \times 5$ array is shown here) can be modeled as follows:

$$
\sum_{i=1}^{m} \sum_{j=1}^{n} X_{i j k}=1-\sum_{t=1}^{k-1} X_{42 t}= \begin{cases}1 & \text { if } X_{42 t}=1, t \leq k-1 \\ 0 & \text { otherwise }\end{cases}
$$

where $2 \leq k \leq T$.

To simplify the model, an additional virtual cell is added adjacent to the sink. The test stimuli droplet is viewed as being finally stored in this virtual cell after test application. In the running example of Figure 5, the virtual cell $(5,2)$ is added; see Figure 6.

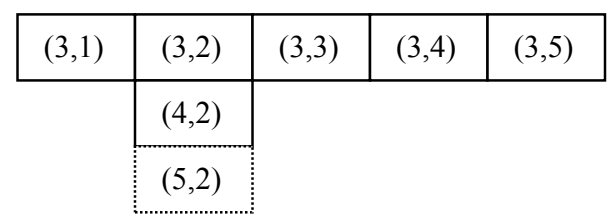

Figure 6: Virtual cell added to the array of Figure 5.

The above constraint can now be expressed as:

a) $\sum_{i=1}^{m} \sum_{j=1}^{n} X_{i j k}=1$, where $1 \leq k \leq T$, and $(i, j)$ is any cell in the microfluidic array, including virtual cell $(5,2)$.

b) $X_{52 k}=\sum_{t=1}^{k-1} X_{42 t}$, where $2 \leq k \leq T$.

(3) Starting point:

This is determined by the location of the droplet source. In our example, $X_{131}=1$, i.e., the cell $(1,3)$ is visited by the test stimuli droplet at time slot 1 .

(4) Movement rules:

When the test stimuli droplet moves in the microfluidic array, it should obey the movement rules described as follows. It only can move to its neighbors i.e., if the droplet visits cell $(i, j)$ at time slot $k$, then for time slot $k+1$, the droplet can only move to column $j+1, j$ or $j-1$ when it remains in the same row $i$. Similarly, if it stays in the same column $j$, the possible rows in time slot $k+1$ are $i-1, i$, or $i+1$.

This rule can be modeled as follows:

Let $P_{k}=\sum_{i=1}^{m} \sum_{j=1}^{n} i \times X_{i j k}$ be the number of the row visited at time-slot $k$. Likewise, let $q_{k}=\sum_{i=1}^{m} \sum_{j=1}^{n} j \times X_{i j k}$ be the umber of the column visited at time-slot $k$. Let $\Delta P_{k}=\left|P_{k+1}-P_{k}\right|$ and $\Delta q_{k}=\left|q_{k+1}-q_{k}\right|$. We must ensure that $\Delta P_{k}+\Delta q_{k} \leq 1$.

We have now developed the ILP model for OSP using Figure 5 as a running example. The general ILP model for an $m \times n$ array is shown in Figure 7, where cell $(a, b)$ refers to the droplet sink and cell $(c, d)$ is adjacent to the droplet source. The complexity of this model is $\mathrm{O}(m n T)$ in the number of variables and $\mathrm{O}(m n+T)$ in the number of 
constraints for an $m \times n$ array. The result obtained using lpsolve for Figure 5 is shown in Table 1. The optimal test schedule for this $3 \times 5$ array generated by lpsolve is shown in Figure 8, where the number in the cell represents the time slot. We notice that some cell, e.g., cells $(2,3)$ and $(2,4)$ in Figure 5 , need to be visited more than once by the test stimuli droplet.

minimize: $C=\sum_{k=1}^{T} k \times X_{a b k}$.
Subject to:
1) $\sum_{k=1}^{T} X_{i j k} \geq 1$, for any cell $(i, j)$ available for testing.
2) $\sum_{k=1}^{T} X_{i j k}=0$, for any cell $(i, j)$ that is running biomedical
assays and not available for testing.
3) $\sum_{k=1}^{T} X_{a b k}=1$, for the sink, i.e., the cell $(a, b)$
4) $\sum_{i=1}^{m} \sum_{j=1}^{n} X_{i j k}=1$, where $1 \leq k \leq T$, and $(i, j) i$ any cell in the
microfluidic array, including virtual cell $\left(a^{*}, b\right)$.
5) $X_{a \cdot b k}=\sum_{t=1}^{k-1} X_{a b t}$, where $2 \leq k \leq T$.
6) $X_{c d 1}=1$
7) $P_{k}=\sum_{i=1}^{m} \sum_{j=1}^{n} i \times X_{i j k} q_{k}=\sum_{i=1}^{m} \sum_{j=1}^{n} j \times X_{i j k}$
8) $\Delta P_{k}=\left|P_{k+1}-P_{k}\right| \Delta q_{k}=\left|q_{k+1}-q_{k}\right|$
9) $\Delta P_{k}+\Delta q_{k} \leq 1$

Figure 7: Integer linear programming (ILP) for a $m \times n$ array.

Table 1: Optimization result for OSP for the example of Figure 5 (Only variables assigned the value 1 are listed).

\begin{tabular}{|l|l|l|l|}
\hline Variables & Value & Variables & Value \\
\hline $\begin{array}{l}\text { Objective } \\
\text { function }\end{array}$ & 13 & $X_{247}$ & 1 \\
\hline $\boldsymbol{C}$ & 13 & $X_{238}$ & 1 \\
\hline$X_{131}$ & 1 & $X_{229}$ & 1 \\
\hline$X_{232}$ & 1 & $X_{2110}$ & 1 \\
\hline$X_{243}$ & 1 & $X_{3111}$ & 1 \\
\hline$X_{254}$ & 1 & $X_{3212}$ & 1 \\
\hline$X_{355}$ & 1 & $X_{4213}$ & 1 \\
\hline$X_{346}$ & 1 & $X_{5214}$ & 1 \\
\hline
\end{tabular}

The ILP model for OSP can easily be extended to find an optimal test schedule for more than one source and more than one sink as follows:

We modify the binary variable $X_{i j k}$ to $X_{i j k l}$ as follows:

$$
X_{i j k l}= \begin{cases}1 & \begin{array}{l}
\text { if cell }(i, j) \text { is visited by test stimuli droplet } \\
l \text { at time slot } k
\end{array} \\
0 & \text { otherwise }\end{cases}
$$

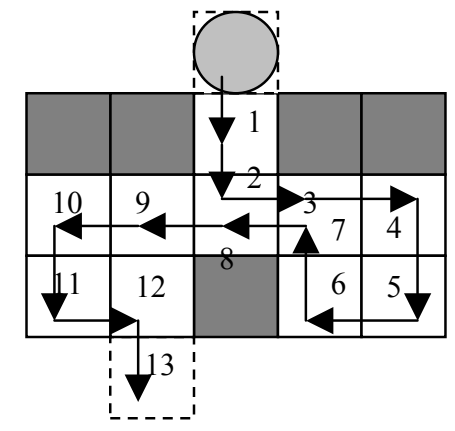

Figure 8: Optimal test schedule for the example of Figure 5.

where $1 \leq k \leq T, 1 \leq l \leq N$, and $N$ is the number of source-sink pairs. $(N=2$ in example of two droplet sources and two droplet sinks; see Figure 9). This ILP model is similar to the above model for OSP, with the following differences:

(1) The objective function is modified to minimize the maximum value of the time when each test stimuli droplet reaches its sink. For the example of Figure 9,

$$
C=\max \left\{\sum_{k=1}^{T} k \times X_{42 k 1}, \sum_{k=1}^{T} k \times X_{42 k 2}\right\}
$$

(2) An additional constraint is incorporated as follows. Any cell $(i, j)$ in the array cannot be visited by more than one testing droplet at the same time slot, i.e., $\sum_{l=1}^{N} X_{i j k l} \leq 1$ where $1 \leq k \leq T$. In the example of Figure 9, this is expressed as: $X_{i j k 1}+X_{i j k 2} \leq 1$.

(3) When multiple test stimuli droplets are applied, each droplet can never be in a cell directly adjacent or diagonally adjacent to another droplet. This restriction prevents two droplets from mixing together. It can be expressed as: a)

$$
\begin{gathered}
\left.P_{k l}=\sum_{i=1}^{m} \sum_{j=1}^{n} i \times X_{i j k l} ; q_{k l}=\sum_{i=1}^{m} \sum_{j=1}^{n} j \times X_{i j k l} \quad l=1,2 ; \mathrm{b}\right) \\
\Delta P_{k 12}=\left|P_{k 2}-P_{k 1}\right| ; \Delta q_{k 12}=\left|q_{k 2}-q_{k 1}\right| ; \text { c) } \Delta P_{k 12} \geq 2,
\end{gathered}
$$$$
\text { or } \Delta q_{k 12} \geq 2 \text {. }
$$

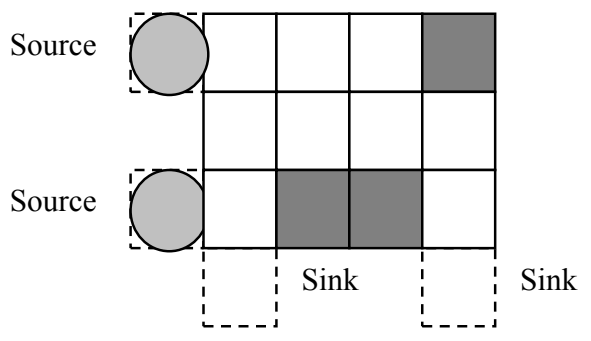

Figure 9: Example of an array with two droplet sources and two droplet sinks. 
The optimized result obtained using lpsolve for the example of Figure 9 is listed in Table 2. The test plan and the droplet paths based on the output of lpsolve are shown in Figure 10.

Table 2: Optimization result for OSP for the example of Figure 9 (Only variables assigned the value 1 are listed).

\begin{tabular}{|l|l|l|l|}
\hline Variable & Value & Variable & Value \\
\hline $\begin{array}{l}\text { Objective } \\
\text { function }\end{array}$ & 8 & $X_{4181}$ & 1 \\
\hline C & 8 & $X_{1112}$ & 1 \\
\hline$X_{3111}$ & 1 & $X_{1222}$ & 1 \\
\hline$X_{3121}$ & 1 & $X_{1332}$ & 1 \\
\hline$X_{2131}$ & 1 & $X_{2342}$ & 1 \\
\hline$X_{2141}$ & 1 & $X_{2452}$ & 1 \\
\hline$X_{2251}$ & 1 & $X_{3462}$ & 1 \\
\hline$X_{2161}$ & 1 & $X_{4472}$ & 1 \\
\hline$X_{3171}$ & 1 & $X_{5482}$ & 1 \\
\hline & & & \\
\hline
\end{tabular}

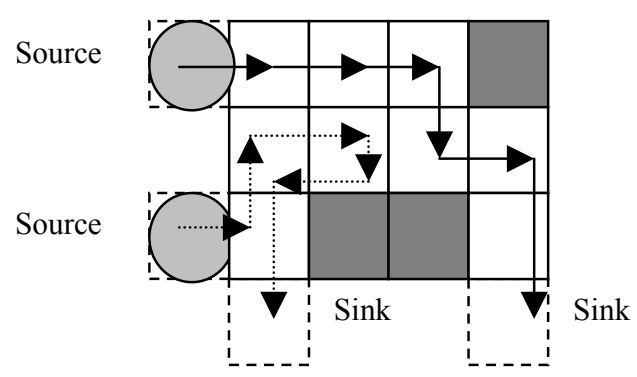

Figure 10: Droplet flow path for the example of Figure 9.

Next we describe how we can modify the ILP model for OSP to derive a test plan that can support concurrent testing during the execution of a biomedical assay. Note that the cells that are used in a biomedical assay, and viewed as unavailable cells in a particular time period, may still be available for testing in another time period. Let $U_{k}$ be the set of cells, where each such cell is denoted by the pair $(i, j)$, used by the assay in time slot $k$. Instead of setting $\sum_{k=1}^{T} X_{i j k l}=0$ for these cells for $1 \leq k \leq T$, we set $\sum_{(i, j) \in U_{k}} X_{i j k l}=0$ for every time slot $k$. In the following section, we consider the test planning problem for a reallife example of and apply the modified ILP model to obtain a concurrent test plan. In the previous models, the cells used in biomedical assays were considered to be unavailable for testing at all times. In this way, we have to use multiple sources and multiple sinks for testing, because the available microfluidic cells are partitioned into many disconnected parts by these unavailable cells. However, based on the modified ILP model of OSP, we can use a single source and a single sink to test this array. Moreover, all microfluidic cells in this array, including those that are used for the biomedical assay, can be tested.

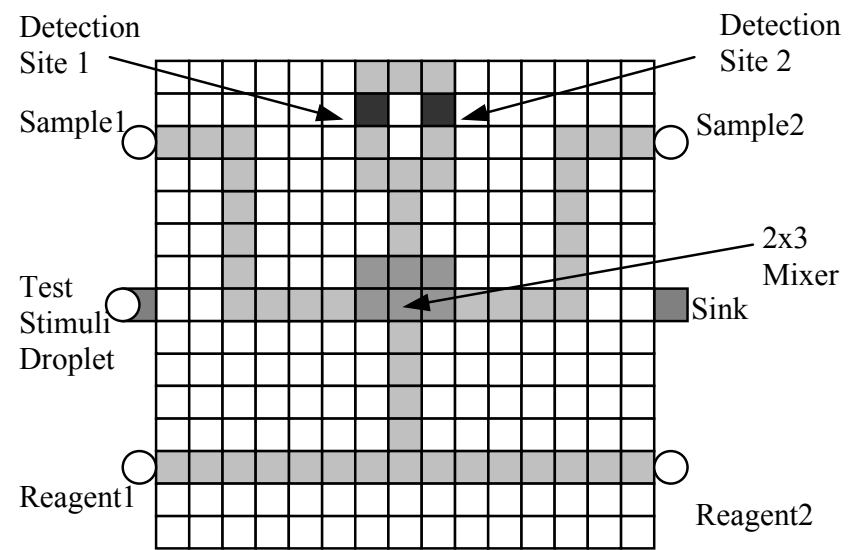

Figure 11: Microfluidic array used for multiplexed biomedical assay.

\section{Example: Concurrent Testing for Multiplexed Biomedical Assay}

In this section, we use a real-life example of a multiplexed biomedical assay to illustrate how the ILPbased scheduling method can be used for the test planning and test resource optimization. The multiplexed biomedical assay in the experiment consists of a glucose assay and a lactate assay based on colorimetric enzymatic reactions, which have been described in Section 2.

The system used for this multiplexed biomedical assay is shown in Figure 11. The fabricated prototype is shown in Figure 3. In this system, the sample droplet containing glucose and the reagent droplet consisting of glucose oxidase, peroxidase, 4-AAP and TOPS are dispensed into a $15 \times 15$ microfluidic array $(22.5 \mathrm{~mm} \times 22.5 \mathrm{~mm})$ from onchip reservoirs. They are guided through the transportation paths, denoted by gray cells, when a $50 \mathrm{~V}$ actuation voltage with the frequency of $16 \mathrm{~Hz}$ is applied to control electrodes. Droplets of the sample and the reagent are transported toward a mixer with a linear array design to mix together. In this $2 \times 3$ array mixer, the mixed droplet turns around two pivot points with a translational step in between; see Figure 12. In experiments, an average mixing time of 6 seconds was achieved at $16 \mathrm{~Hz}$ by rotating the droplet counter-clockwise in the $2 \times 3$ array [28].

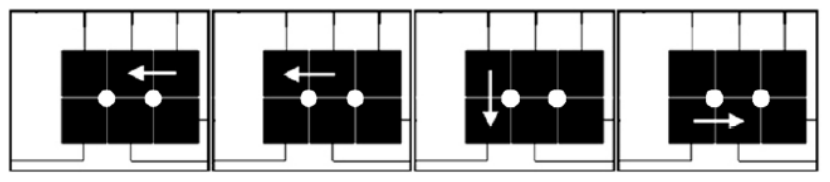

Figure 12: Motion in a $2 \times 3$ mixer array.

The enzymatic reactions are carried out during the mixing step. After this step, a droplet containing the product of the reaction, such as colored quinoneimine, is moved to the optical detection site 1 , where the absorbance is measured for about 13 seconds using the 
LED-photodiode setup described in Section 2. Finally, the droplet leaves the microfluidic array to the waste reservoir. In this experiment, filler fluid of immiscible $1 \mathrm{cSt}$ silicone oil is used to surround the droplet to prevent evaporation and reduce the droplet actuation voltage. A similar procedure is also used in the lactase assay. These assays can be integrated on a microfluidic system to form a multiplexed biomedical assay for clinical diagnosis on metabolites. The schedule of this multiplexed assay is shown in Table 3. In order to detect catastrophic faults in this system, such as electrode degradation [17], during field operation, we add a built-in test hardware to this system. The test hardware consists of droplet sources that generate and dispense the test stimuli droplet (e.g. 0.1M KCL solution), and droplet sinks connected to an on-chip capacitive detection circuit. The goal of the concurrent testing is to ensure that the test stimuli droplet traverses every cell in this $15 \times 15$ array, i.e., not only the spare cells, but also the cells used in the biomedical assay.

Table 3: Schedule of multiplexed biomedical assay.

\begin{tabular}{|c|c|}
\hline $\begin{array}{l}\text { Time } \\
\text { (second) }\end{array}$ & Operation \\
\hline 0 & $\begin{array}{l}\text { Sample } 2 \text { and reagent } 2 \text { start to move towards the } \\
\text { mixer. }\end{array}$ \\
\hline 0.8 & $\begin{array}{l}\text { Sample } 2 \text { and reagent } 2 \text { begin to mix together and } \\
\text { turn around in the } 2 \times 3 \text { array }\end{array}$ \\
\hline 6.0 & $\begin{array}{l}\text { (1) Sample } 1 \text { and reagent } 1 \text { start to move } \\
\text { towards the mixer. } \\
\text { (2) Sample } 2 \text { and reagent } 2 \text { continue the } \\
\text { mixing. }\end{array}$ \\
\hline 6.8 & $\begin{array}{l}\text { (1) Sample } 2 \text { and reagent } 2 \text { finish the mixing } \\
\text { and product } 2 \text { leave the mixer to optical } \\
\text { detection location } 2 \text {. } \\
\text { (2) Sample } 1 \text { and reagent } 1 \text { begin to mix in } \\
2 \times 3 \text { array mixer. }\end{array}$ \\
\hline 12.8 & $\begin{array}{l}\text { (1) Sample } 1 \text { and reagent } 1 \text { finish the mixing } \\
\text { and product } 1 \text { leave the mixer to the } \\
\text { optical detection location } 1 . \\
\text { (2) Product } 2 \text { continues the absorbance } \\
\text { detection. }\end{array}$ \\
\hline 19.8 & $\begin{array}{l}\text { (1) Product } 2 \text { finishes optical detection and } \\
\text { leaves the array to the waste reservoir. } \\
\text { (2) Product } 1 \text { continues the absorbance } \\
\text { detection. }\end{array}$ \\
\hline 25.8 & $\begin{array}{l}\text { Product } 1 \text { finishes optical detection and leaves the } \\
\text { array to the waste reservoir. One procedure of the } \\
\text { multiplexed biomedical assay ends. }\end{array}$ \\
\hline
\end{tabular}

Now we apply a modified integer linear programming (ILP) model to the above example to derive an optimal test plan. Due to the inherent complexity of this optimization problem, the computational effort required by the ILP model increases dramatically for a microfluidic array of large size. For this example of a $15 \times 15$ array, we therefore modify the ILP method as follows. We first partition the large array into non-overlapping parts. Thus, a new array of the smaller size is formed, where each new cell represents a partition. For example, we can partition

Paper 32.1

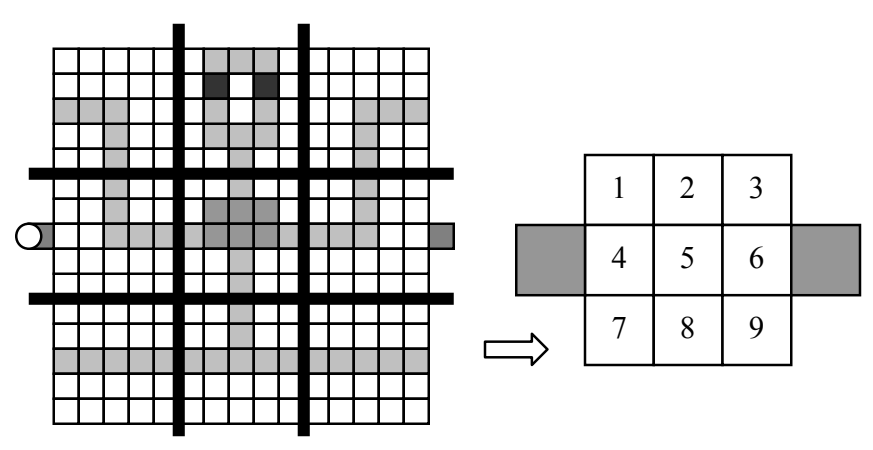

Figure 13: Partition of a $15 \times 15$ array.

the $15 \times 15$ array in the example into nine non-overlapping parts, and the overall system can now be viewed as a new $3 \times 3$ array; see Figure 13. Each partition, i.e., the cell of the new small size array, consists of $5 \times 5$ microfluidic cells. We then define the following operations that can be performed in the partition:

1) Transportation: sample droplets and reagent droplets move through one partition along five grid points (e.g., in cells $1,3,4,6,7,8,9$ ). This operation takes 0.3 second, when a control voltage with the frequency of 16 $\mathrm{Hz}$ is applied.

2) Mixing: sample droplets mix with reagent droplets, and they move round a $2 \times 3$ array to accelerate the mixing procedure (e.g., in cell 5). The operation time is 6 seconds.

3) Optical detection: the absorbance of the droplet containing the colorimetric product of enzymatic reaction is detected by the LED- photodiode (e.g., in cell 2). The detection for an assay product takes 13 seconds.

4) Testing: the test stimuli droplet sweeps all the microfluidic cells in this partition. The optimal testing time cost is 1.7 second, during which the test stimuli droplet traverses all 25 cells when a control voltage with the frequency of $16 \mathrm{~Hz}$ is applied.

We next apply the ILP scheduling model described in Section 5 to the $3 \times 3$ array obtained from the partitioning. We first modify the time-slot. The length of a time slot equals to the operation time of testing for one cell in the $3 \times 3$ array, i.e., 1.7 second. In this way, we digitize the operation schedule of the multiplexed biomedical assay. When a partition cell is used in the operation of transportation, mixing, or optical detection during some time-slot, this cell is considered to be unavailable in this time-slot; see Table 4. Note that, except for the testing operation, each partition cell has a segregation region, which wraps round the functional operation region. This isolates the droplet operated in one partition from the droplets in the adjacent partitions. This implies that the test stimuli droplet can be traversing one partition, while the biomedical assay is being carried out in the adjacent partition. We then replace the inequality in the previous 
[9] A. K Henning, "Microfluidic MEMS", Proc. IEEE Aerospace Conference, vol. 1 pp. 471 - 486, 1998.

[10] E. Verpoorte and N. F. De Rooij, "Microfluidics meets MEMS", Proceedings of the IEEE, vol. 91, pp. $930-953,2003$.

[11] M. G. Pollack, R. B. Fair and A. D. Shenderov, "Electrowetting-based actuation of liquid droplets for microfluidic applications", Applied Physics Letters, vol. 77, pp. 1725-1726, 2000.

[12] S. K. Cho, S. K. Fan, H. Moon and C. J Kim, "Toward digital microfluidic circuits: creating, transporting, cutting and merging liquid droplets by electrowetting-based actuation", Proc. IEEE Conf. MEMS, pp. 32-52. 2002.

[13] V. Srinivasan, V. K. Pamula, M. G. Pollack and R. B. Fair, "A digital microfluidic biosensor for multianalyte detection", Proc. IEEE Conf. MEMS, pp. 327-330, 2003.

[14] V. Srinivasan, V. K. Pamula, M. G. Pollack and R. B. Fair, "Clinical diagnostics on human whole blood, plasma, serum, urine, saliva, sweat, and tears on a digital microfluidic platform", Proc. $\mu T A S$, pp. 1287-1290, 2003.

[15] M. G. Pollack, P. Y. Paik, A. D. Shenderov, V. K. Pamula, F. S. Dietrich and R. B. Fair, "Investigation of electrowetting-based microfluidics for real-time PCR applications", Proc. $\mu$ TAS, pp. 619-622, 2003.

[16] International Technology Roadmap for Semiconductor (ITRS), http://public.itrs.net/Files/2001ITRS/Test.pdf.

[17] F. Su, S. Ozev and K. Chakrabarty, "Testing of droplet-based microelectrofluidic systems", Proc. IEEE Int. Test Conf., pp. 1192-1200, 2003.

[18] Videos on droplet transport, dispensing, mixing and biomedical assays [Online]. Available at http://www.ee.duke.edu/Research/microfluidics

[19] M. G. Pollack, A. D. Shenderov and R. B. Fair, "Electrowetting-based actuation of droplets for integrated microfluidics", Lab on a Chip, vol. 2, pp. 96-101, 2002.

[20] S. Mir, B. Charlot and B. Courtois. "Extending faultbased testing to microelectromechanical systems." Journal of Electronic Testing : Theory and Applications, vol. 16, pp. 279-288, 2000.

[21] A. Kolpekwar and R. D. Blanton, "Development of a MEMS testing methodology", Proc. IEEE Int. Test Conf., pp. 923-93, 1997.

[22] N. Deb and R. D. Blanton, "Analysis of failure sources in surface-micromachined MEMS", Proc. IEEE Int. Test Conf., pp. 739-749, 2000.

[23] H. G. Kerkhoff, "Testing philosophy behind the micro analysis system", Proc. SPIE: Design, Test and Microfabrication of MEMS and MOEMS, vol.
3680, pp.78-83, 1999.

[24] H. G. Kerkhoff and H. P. A. Hendriks, "Fault modeling and fault simulation in mixed micro-fluidic microelectronic systems", Journal of Electronic Testing: Theory and Applications, vol. 17, pp. 427437, 2001.

[25] H. G. Kerkhoff and M. Acar, "Testable design and testing of micro-electro-fluidic arrays", Proc. IEEE VLSI Test Symposium, pp. 403-409, 2003.

[26] R. Graham, E. Lawler, J. Lenstra and A. R. Kan, "Optimization and approximation in deterministic sequencing and scheduling: A survey", Annals of Discrete Mathematics, vol. 5, pp. 287-326, 1979.

[27] M. Berkelaar. lpsolve. Eindhoven Univ. Technol., Eindhoven, The Netherlands [Online]. Available at ftp://ftp.ics.ele.tue.nl/pub/lp_solve.

[28] P. Paik, V. K. Pamula and R. B. Fair, "Rapid droplet mixers for digital microfluidic systems", Lab on a Chip, vol. 3, pp. 253-259, 2003. 